\title{
TNF regulates transcription of NLRP3 inflammasome components and inflammatory molecules in cryopyrinopathies
}

\author{
Matthew D. McGeough, ${ }^{1}$ Alexander Wree, ${ }^{1,2}$ Maria E. Inzaugarat, ${ }^{2}$ Ariela Haimovich, ${ }^{1}$ Casey D. Johnson, ${ }^{1}$ Carla A. Peña, ${ }^{1}$ \\ Raphaela Goldbach-Mansky, ${ }^{3}$ Lori Broderick, ${ }^{1}$ Ariel E. Feldstein, ${ }^{1}$ and Hal M. Hoffman ${ }^{1}$ \\ 'Department of Pediatrics, UCSD, La Jolla, California, USA. ²Department of Internal Medicine III, RWTH University Hospital Aachen, Cermany. ${ }^{3}$ National Institute of Allergy and Infectious Diseases (NIAID) \\ NIH, Bethesda, Maryland, USA
}

\begin{abstract}
The NLRP3 inflammasome is a protein complex responsible for caspase-1-dependent maturation of the proinflammatory cytokines IL-1ß and IL-18. Gain-of-function missense mutations in NLRP3 cause the disease spectrum known as the cryopyrin-associated periodic syndromes (CAPS). In this study, we generated NIrp3-knockin mice on various KO

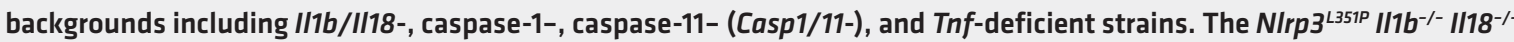
mutant mice survived and grew normally until adulthood and, at 6 months of age, exhibited marked splenomegaly and leukophilia. Injection of these mice with low-dose LPS resulted in elevated serum TNF levels compared with NIrp3 ${ }^{\text {L351P }}$

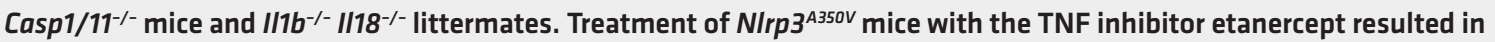
all pups surviving to adulthood, with normal body and spleen/body weight ratios. NIrp $3^{A 350 V} \mathrm{Tnf}^{-/-}$mice showed a similar phenotypic rescue, with marked reductions in serum IL-1 $\beta$ and IL-18, reduced myeloid inflammatory infiltrate in the skin and spleen, and substantial decreases in splenic mRNA expression of both inflammasome components (NIrp3, Pycard, pro-Casp1) and pro-cytokines (I/1b, I/18). Likewise, we observed a reduction in the expression of both pro-Casp1 and pro-II1b in cultured $\mathrm{NIrp3}^{\mathrm{A350V}} \mathrm{Tnf}^{-/-} \mathrm{BM}$-derived DCs. Our data show that TNF is an important transcriptional regulator of NLRP3 inflammasome components in murine inflammasomopathies. Moreover, these results may have therapeutic implications for CAPS patients with partial responses to IL-1-targeted therapies.
\end{abstract}

\section{Introduction}

The inflammasome complex is formed by NLRP3, along with the adaptor protein pycard (ASC) and pro-caspase-1, which, when activated by various endogenous and microbe-derived danger signals, directs the autocleavage and processing of procaspase-1 (1). Activated caspase- 1 cleaves both pro-IL-1 $\beta$ and pro-IL-18 to their mature, secretory forms but has also been shown to result in pyroptosis, a proinflammatory form of programmed cell death $(2,3)$. NLRP3 has been implicated in the pathogenesis of numerous human diseases including gout, atherosclerosis, and Alzheimer's disease (4).

Gain-of-function missense mutations in NLRP3 cause the autoinflammatory disease spectrum known as the cryopyrinassociated periodic syndromes (CAPS), characterized by inflammatory symptoms involving skin, muscles, joints, conjunctiva, and the CNS (5). CAPS represent a continuum in disease severity, with

Related Commentary: p. 4235

Authorship note: M.D. McGeough and A. Wree contributed equally to this work Conflict of interest: R. Goldbach-Mansky has received grant support from Sobi Biovitrum, Regeneron, Novartis, and Eli Lilly and Company. H.M. Hoffman is a consultant for Regeneron, Novartis, and Sobi Biovitrum and has received research support from GlaxoSmithKline.

Submitted: September 19, 2016; Accepted: September 21, 2017

Reference information: J Clin Invest. 2017;127(12):4488-4497.

https://doi.org/10.1172/JC190699. neonatal-onset multisystem inflammatory disease (NOMID) at the severe end, characterized by continuous inflammatory symptoms with accompanying neurological impairment and deforming arthropathy; the intermediate Muckle-Wells syndrome (MWS), with recurrent inflammatory episodes and a risk of long-term sequelae such as hearing loss and amyloidosis; and familial cold autoinflammatory syndrome (FCAS) at the mild end, with inflammatory symptoms often associated with generalized cold exposure (6). IL-1 blockade has been largely successful in the treatment of CAPS, however, there are increasing reports of incomplete clinical responses (our unpublished clinical observations), emphasizing the need for further understanding of the disease mechanisms and novel drug development.

Knockin mouse models of Nlrp3 inflammasomopathies have served as important and translatable tools to understand CAPS disease pathogenesis, demonstrating that disease is primarily driven by the innate immune system and specifically by inflammatory cells of myeloid cell lineage $(7,8)$. As in human CAPS, IL-1 $\beta$ has been shown to play a major role in CAPS disease pathogenesis in mice, however, additional mechanisms appear to be involved (9). Clear and independent roles were previously defined for IL-1 and IL-18 signaling in disease progression using KO mice deficient in Illr or Il18r. However, when both cytokine pathways were disrupted using double-KO mice for Il1r and Il18, a substantial disease phenotype remained, suggesting potential contributions from pyroptosis or other NLRP3 inflammasome-driven mechanisms (9). 


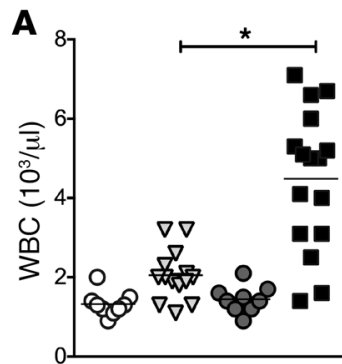

D
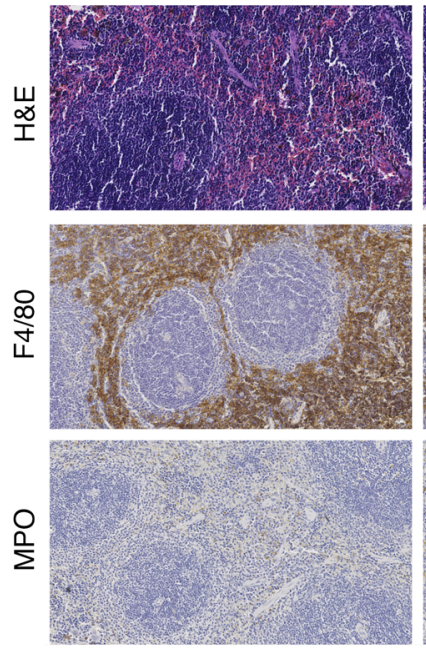

B

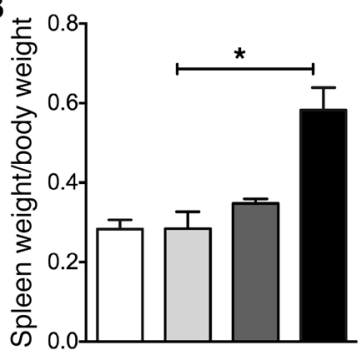

NIrp3 ${ }^{\text {L351P }}$ Casp1/11/--
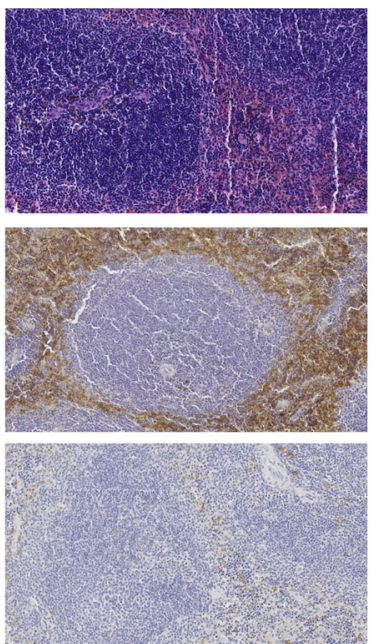

C

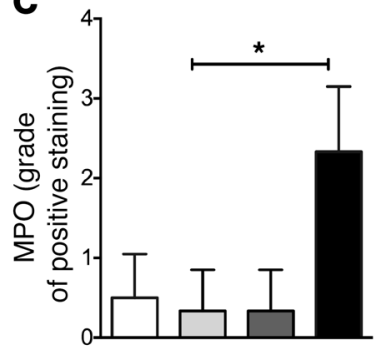

$\left\|1 b^{-1-}\right\| 18^{-1-}$
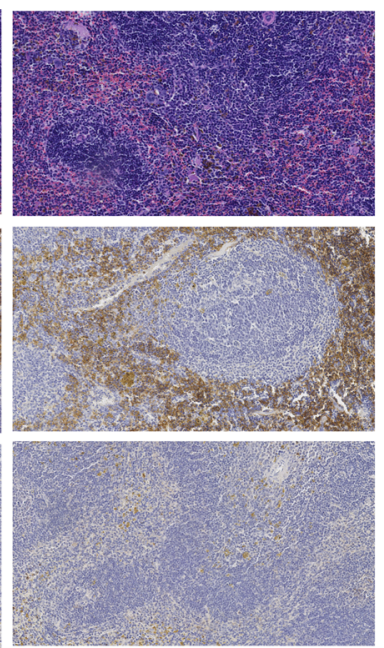

$\square$ Casp $1 / 11^{-1}$

$\square$ NIrp3 $3^{L 351 P}$ Casp1/11-

$111 b^{-/} \| 18^{-1-}$

NIrp3 $3^{L 351 P}\left\|11 \mathrm{~b}^{-1-}\right\| 18^{-1-}$

Figure 1. Role for caspase-1-dependent inflammatory mediators other than IL-1 $\beta$ and IL-18 in NIrp3-mutant mice. NIrp $3^{L 351 P}\left\|/ 1 b^{-/-}\right\| / 18^{-/-}$mice had (A) elevated WBC numbers (each data point represents an individual mouse) and (B) splenomegaly, as evidenced by spleen weight/body weight ratios com-

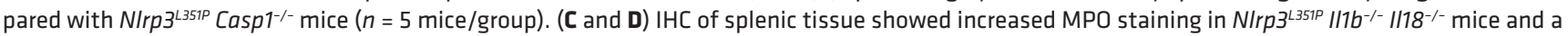
similar degree of F4/80 staining in all groups. Images are representative of 6 mice per group (original magnification, $\times 20$ ). Scale bar: $100 \mu \mathrm{m}$. ${ }^{*} P<0.05$, by

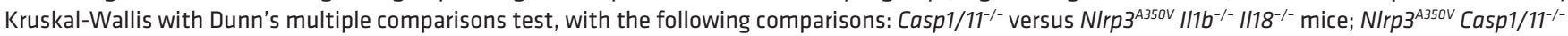

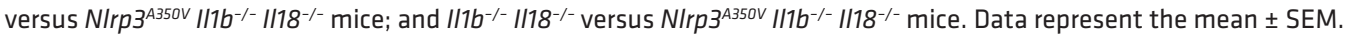

To further elucidate systemic autoinflammatory disease mechanisms other than IL-1 $\beta$ and IL-18, we generated Nlrp3 $3^{2351 P}$ $I l 1 b^{-/} I l 18^{-/}$mice. Phenotypic characterization of these mice at 6 months of age or older revealed systemic inflammation dependent on caspase-1 and/or caspase-11 and a marked elevation in serum TNF levels following in vivo LPS challenge. This finding prompted a more in-depth evaluation of TNF in our CAPS mouse models. Treatment of $N \operatorname{lrp} 3^{\mathrm{A} 350 \mathrm{~V}}$ mice with the TNF inhibitor etanercept or breeding onto a $T n f^{\prime-}$ - background provided phenotypic rescue. Our results indicate that TNF plays an unexpected and significant role in murine inflammasomopathies and suggest the existence of another important mediator in the development and progression of these autoinflammatory disorders.

\section{Results}

We previously showed that NLRP3 inflammasome-mediated IL-1 $\beta$, IL-18, and cell death play distinct spatial and temporal roles in the pathogenesis of CAPS (9). In order to further elucidate nonIL-1 $\beta / \mathrm{IL}-18$-dependent disease mechanisms, we generated Cre lysozyme (LysM-Cre) conditional myeloid-restricted $\mathrm{Nlrp} 3^{\mathrm{LS51P} /+}$ mutant mice on a combined Il1b/Il18-deficient background

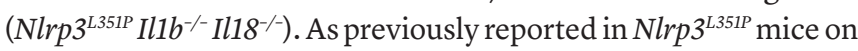
an Illr/-Il18 ${ }^{-/}$double-KO background, we observed a partial phe- notypic rescue in the $\mathrm{Il1b}^{-1-} \mathrm{Il18^{-/ }}$ background that was substantially better than that of the mutant mice on each of the cytokine-KO backgrounds alone. We then studied surviving mice beyond 6 months of age and found persistent, chronic systemic inflammation that was evidenced by a significant elevation in WBC counts as compared with those of caspase-1/caspase-11-deficient Nlrp $3^{\text {L35IP }}$

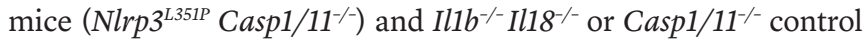
animals (Figure 1A). Analysis of spleens from these mice revealed marked splenomegaly and a significant neutrophilic infiltrate, as shown by immunohistologic staining for myeloperoxidase (MPO), which was not present in Nlrp $3^{L 351 P}$ Casp1/11/- or control mice (Figure $1, \mathrm{~B}-\mathrm{D})$. We observed no changes in macrophage numbers or distribution or other pathologic changes in the spleens from these mice. This implicates an NLRP3 inflammasome- and caspase-1and/or caspase-11-mediated proinflammatory pathology that is independent of both IL-1 $\beta$ and IL-18.

To further examine the mechanisms driving disease in CAPS mice, we attempted to exaggerate the NLRP3 inflammasomemediated inflammatory response by challenging the groups of mice described above in a sublethal endotoxic shock model. We injected LPS i.p. and observed the mice over the next 24 hours for survival and measured serum cytokine levels. Survival analysis of mice injected i.p. with LPS $(10 \mu \mathrm{g} / \mathrm{g})$ showed that $80 \%$ of the 
A
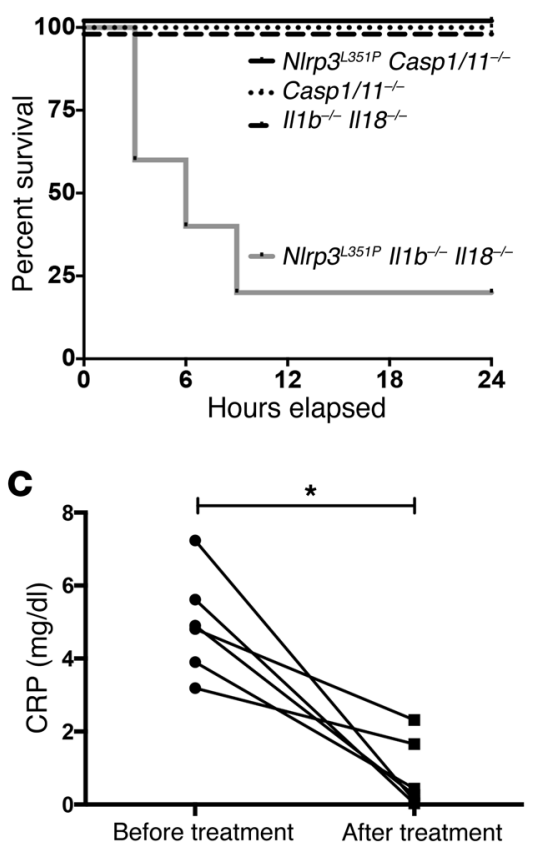

B $\triangle$ Casp $1 / 11^{-/} \quad 0\left\|11 b^{-/}\right\| 18^{-/-} \quad$ WT

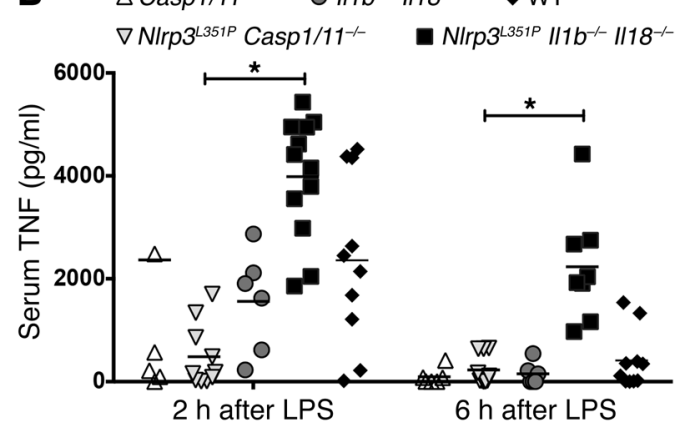

D

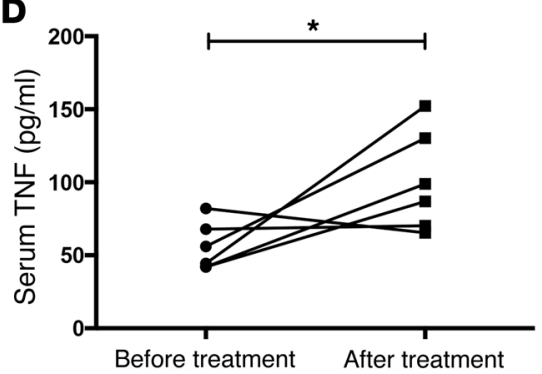

Figure 2. TNF is elevated in NIrp $3^{L 351 P}\left\|1 b^{-/-}\right\| 18^{-/-}$mice following stimulation with LPS and in the serum of patients treated with an IL-1 receptor antagonist. (A) Survival of NIrp $3^{L 351 P} / 11 \mathrm{~b}^{-1-} 1 / 18^{-/-}$mice was markedly decreased following a nonlethal dose of LPS $(10 \mu \mathrm{g} / \mathrm{g})(n=5$ mice/group). (B) Serum TNF levels were

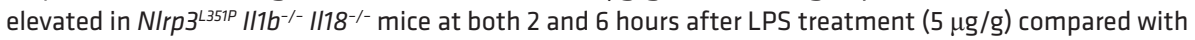
levels in NIrp3 ${ }^{L 351 P}$ Casp1 $^{-1-}$ mice (horizontal bar represents the mean). (C) Levels of serum CRP were significantly reduced in pediatric NOMID patients after treatment with anakinra. (D) Following treatment, serum TNF levels were significantly elevated in patients compared with their baseline levels. Each data point represents an individual mouse or patient. ${ }^{*} P<0.05$, by Kruskal-Wallis with Dunn's multiple comparisons test (B) or 1-tailed Student's $t$ test (C and D).

Nlrp3 $3^{L 351 P} \mathrm{Il} \mathrm{b}^{-/-} \mathrm{Il18^{-/ }}$ mice died within 9 hours of injection and $100 \%$ of the control and Nlrp $3^{L 351 P}$ Casp $1 / 11^{-/-}$mice survived (Figure $2 \mathrm{~A})$. We then injected a nonlethal dose of LPS $(5 \mu \mathrm{g} / \mathrm{g})$ and performed ELISA to measure serum levels of the proinflammatory cytokines IL-6, IL-1 $\alpha$, IL-17, KC (also called IL-8), and TNF at baseline and 2 and 6 hours after injection. We observed no significant differences in serum IL-6, IL-1 $\alpha$, IL-17, or KC levels between

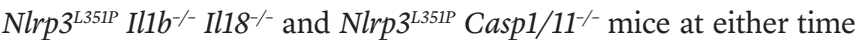
point (Supplemental Figure 1; supplemental material available online with this article; https://doi.org/10.1172/JCI90699DS1). Baseline values for TNF were undetectable in all groups (data not shown), however a marked elevation of serum TNF levels was noted 2 and 6 hours after LPS injection in Nlrp3 $3^{L 351 P} \mathrm{Illb}^{-/-} \mathrm{Il18^{-/- }}$ mice but was not observed in the control or $N \operatorname{lrp} 3^{L 351 P}$ Casp1/11 $11^{-/}$animals (Figure 2B). These results suggest that LPS drives TNF in serum in these mice in a NLRP3 inflammasome- and caspase-1- and/or caspase-11-dependent, but IL-1 $\beta$ - and IL-18-independent, manner.

We next investigated whether our findings in CAPS mice are translatable to human CAPS patients. Baseline and post-treatment measurements of serum cytokine levels were collected from a longitudinal study of NOMID patients on anakinra (IL-1 receptor antagonist) (10). IL-1 blockade was successful in reducing CAPS-associated symptoms and chronic inflammation in this group of patients, with all treated patients showing a significant reduction in C-reactive protein (CRP) (Figure 2C). Interestingly, in this cohort, we found that serum TNF levels were elevated in the treated NOMID patients as compared with levels in patients at baseline and in pediatric controls (Figure 2D, and data not shown), similar to what was observed in our LPS-challenged Nlrp3-mutant mice on an Il1b/Il18KO background. This finding suggests that a mutant gain-of-function NLRP3 inflammasome mediates TNF release in the serum of human CAPS patients that is not blocked by IL-1targeted therapy.

To further investigate the role of TNF in NLRP3-mediated disease, we switched from the $N l r p 3^{L 351 P}$ model (since these mice die in the perinatal period) and used our less severe CAPS mouse model (Nlrp $3^{A 350 V}$ LysM$\mathrm{Cre}$ ), in which pups develop systemic inflammation primarily mediated by myeloid cells and perish by day 14 , so that we would have surviving animals as controls (8). Nlrp $3^{A 350 V}$ pups were treated with the TNF inhibitor etanercept beginning on day 2 and ending on day 12 of life. Surprisingly, the treatment normalized growth during the treatment period and prolonged survival for an average of 23 days after cessation of treatment (Figure 3, A-C). The improved survival and growth parameters suggest that targeting TNF has the potential to significantly reduce NLRP3 inflammasome-mediated inflammation, despite the presence of existing systemic inflammatory symptoms (skin abscesses) at birth.

Following the therapeutic success of etanercept, we decided to further study the role of TNF in murine CAPS using a genetic approach. We crossed Nlrp $3^{A 35 O V}$ mice with $T n f^{--}$mice to generate Cre lysozyme-conditional (LysM-Cre-conditional), myeloidrestricted mutant mice on Tnf-KO and heterozygous-KO backgrounds (Nlrp3 $3^{A 35 O V} \mathrm{Tnf}^{-/}$LysM-Cre and Nlrp3 ${ }^{\mathrm{A35OV}} \mathrm{Tnf}^{+/-}$LysMCre, respectively). Survival analysis showed complete rescue for Nlrp $3^{A 35 O V} \mathrm{Tnf}^{-/-}$mice, with all animals surviving to adulthood. In contrast, $N \operatorname{lrp} 3^{A 350 V} \mathrm{Tnf}^{+/-}$animals exhibited a partial rescue, with mice surviving an average of 22 days compared with $N l r p 3^{A 35 O V}$ mice, which died after 9 days on average, suggesting a gene dosage-dependent effect of TNF expression on the disease pathology of CAPS mice (Figure 4A). Furthermore, Nlrp $3^{A 35 O V}$ Tnf $f^{--}$mice had normal phenotypic appearance and growth, in contrast to the skin erythema, hair growth defect, and poor weight gain observed in $N l r p 3^{A 35 O V}$ mice on a Tnf-intact background. Nlrp $3^{A 35 O V} \mathrm{Tnf}^{+/-}$mice showed an intermediate phenotype with respect to skin rash, hair growth, and weight (Figure 4, B and C). Analysis of spleen weights from these animals as a percentage of total body weight revealed marked splenomegaly in the $N \operatorname{lrp} 3^{A 350 V}$ and $N \operatorname{lrp} 3^{A 35 O V} \mathrm{Tnf}^{+/-}$mice, with a significant reduction in the spleen weights of $N \operatorname{lrp} 3^{\mathrm{A} 35 \mathrm{OV}}$ 

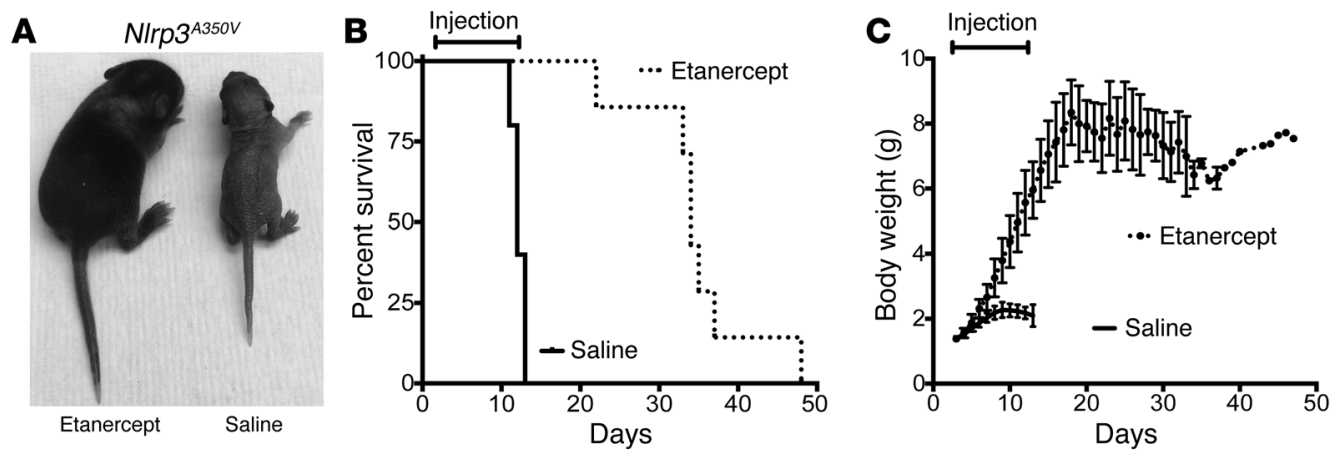

Figure 3. Treatment with etanercept ameliorates systemic inflammatory disease and normalizes growth in NIrp3 $3^{\text {A350V }}$ mice. (A-C) Injection (s.c.) of etanercept every other day, beginning the day after birth, rescued and normalized growth in N/rp3 $3^{A 350 V}$ mice during the treatment period. After stopping treatment, survival was extended for an average of 23 days. $n=7$ for etanercept-treated NIrp $3^{\mathrm{A35OV}}$ mice and $n=5$ for saline-injected $\mathrm{NIrp} 3^{\mathrm{A3} 50 \mathrm{~V}}$ mice.

Tnf $f^{\wedge}$ animals (Figure 4D). Measurement of serum cytokine levels revealed significant decreases in both IL-1 $\beta$ and IL-18 in Nlrp3 $3^{\mathrm{A3} 50 \mathrm{~V}}$ $\mathrm{Tnf}^{\circ} /$ mice as compared with levels in $N \operatorname{lrp} 3^{\mathrm{A35OV}}$ mice (Figure 4, $\mathrm{E}$ and $\mathrm{F})$. Interestingly, serum IL-1 $\beta$ levels were unchanged in Nlrp3 $3^{\mathrm{A3} 5 \mathrm{OV}} \mathrm{Tnf}^{+/}$mice, while we detected an intermediate but significant reduction in serum IL-18 levels, supporting an important gene dosage-dependent regulatory role for TNF with regard to serum IL-18 levels in young mice (Figure 4, E and F).

Skin histology of $N l r p 3^{\mathrm{A3} 5 \mathrm{OV}}$ pups showed a marked inflammatory cell infiltrate in the dermis that primarily consisted of neutrophils and a lack of s.c. fat (Figure 5A). The skin of Nlrp $3^{\mathrm{A} 35 \mathrm{OV}}$ $T n f^{\wedge} /$ mice was indistinguishable from that of $T n f^{\wedge} /$ mice, indicating an important role for TNF in the cutaneous inflammation seen in CAPS mice. $N \operatorname{lrp} 3^{3^{435 O V}} \mathrm{Tnf} f^{+/}$mice again showed an intermediate and variable phenotype, with an intermediate amount of s.c. fat (Figure 5A). Moreover, we found that dermal thickness was increased in the $N \operatorname{lrp} 3^{\mathrm{A} 350 \mathrm{~V}}$ mice and markedly reduced in the Nlrp $3^{\mathrm{A} 350 \mathrm{~V}} \mathrm{Tnf}^{-/}$animals (Figure $5 \mathrm{~B}$ ). IHC of skin sections from $N \operatorname{lrp} 3^{\mathrm{A} 350 \mathrm{~V}}$ mice showed an increased percentage of $\mathrm{F} 4 / 80$-stained areas as well as higher numbers of MPO-positive cells. These pathologic findings were normalized in $\mathrm{Nlrp}^{\mathrm{A35OV}} \mathrm{Tnf}^{-/}$mice and an intermediate phenotype was observed in $N \operatorname{lrp} 3^{\mathrm{A35OV}} \mathrm{Tnf}^{+/-}$ mice (Figure 5, A, C, and D). Quantitative PCR (qPCR) analysis confirmed a prominent macrophage expression primarily of an M1 phenotype, as evidenced by elevated levels of Adgre1 (F4/80) and Nos2 (iNos) but similar levels of Arg1 (M2) in Nlrp $3^{4350 \mathrm{~V}}$ skin compared with that of littermate controls. KO of Tnf significantly reduced both $F 4 / 80$ and $i N o s$, with intermediate levels detected in the Nlrp3 $3^{\mathrm{A35OV}} \mathrm{Tnf}^{+/-}$animals (Supplemental Figure 2). We also noted elevated Cxcl1 and Cxcl 2 expression levels in $N \operatorname{lrp} 3^{\mathrm{A35OV}}$ skin that were markedly reduced in $\mathrm{Nlrp}^{\mathrm{A35OV}} \mathrm{Tnf}^{\prime-}$ skin and slightly reduced in $\mathrm{Nlrp}^{\mathrm{A35OV}} \mathrm{Tnf}^{+/}$skin (Supplemental Figure 2). These results are consistent with macrophage- and possibly mast cellmediated chemokine release, resulting in a neutrophilic infiltrate that is also TNF mediated (11).

Histologic analysis of spleens demonstrated a similar pattern of robust positive staining for MPO in $N \operatorname{lrp} 3^{A 350 V}$ mice, intermediate staining in $\mathrm{Nlrp}^{3 \mathrm{A35OV}} \mathrm{Tnf} \mathrm{f}^{+/}$mice, and notably reduced staining in spleens from Nlrp $3^{\mathrm{A35OV}} \mathrm{Tnf} /$ - mice. Interestingly, we observed no significant decrease in mutant NLRP3-associated F4/80 staining or expression, suggesting that TNF was mainly responsible for the neutrophilic infiltrate in the spleen (Figure 6A). While there was no difference in the total number of macrophages in tissue, qPCR analysis of the inflammatory infiltrate in the spleen showed a significant reduction in the expression of $L y 6 c$ and $i N o s$ in Nlrp $3^{A 35 O V}$ Tnf/- mice, indicating a reduction in inflammatory (type 1) macrophages in the spleens from these animals (Figure 6B). Consistent with our histologic observations, the expression of neutrophilic markers, including $\mathrm{Mpo}, \mathrm{Cxcl1}$, and $\mathrm{Cxcl} 2$, was also significantly reduced in Nlrp3 $3^{\mathrm{A3} 5 \mathrm{OV}} \mathrm{Tnf}^{\wedge} /$ mice (Figure 6, B and C). We also measured splenic mRNA expression of inflammasome components and found significant reductions in the expression of Nlrp3, Asc, and pro-Casp1 in Nlrp $3^{A 350 V} \mathrm{Tnf} /-$ mice, with similar decreases seen in expression of the pro-cytokines IL-1 $\beta$ and IL-18 in spleens from these mice compared with those from $\mathrm{Nlrp}^{\mathrm{A} 350 \mathrm{~V}}$ animals. Intermediate mRNA expression levels were detected in Nlrp $3^{A 350 \mathrm{~V}}$ $\mathrm{Tnf} f^{+/}$mice compared with levels in Nlrp $3^{\mathrm{A35OV}}$ and $N \operatorname{lrp} 3^{\mathrm{A35OV}} \mathrm{Tnf}^{-{ }^{--}}$ animals (Figure 6, D and E).

To further explore the regulatory role of TNF on inflammasome-mediated disease and rule out the possibility that the observed difference in expression levels of inflammasome components and pro-cytokines in spleens from mutant mice on a TNF-deficient background was not due simply to differences in the number of infiltrating myeloid cells, we cultured BM-derived DCs (BMDCs) from previously described inducible Nlrp3 $3^{A 350 \mathrm{~V}}$ Cre-ERT2 mice (8) and similarly age-matched adult Nlrp3 $3^{A 35 O V}$ Tnf $f^{\prime-}$ mice. We found that the expression levels of pro-Casp1 and pro-Illb mRNA were significantly reduced in Nlrp $3^{A 350 V} \mathrm{Tnf} /$ - cells as compared with levels in Nlrp $3^{\mathrm{A35OV}} \mathrm{Cre-ERT2}$ cells (Figure 7A). Stimulation of BMDCs with $1 \mathrm{ng} / \mathrm{ml}$ LPS elevated the mRNA levels of all genes studied and essentially equalized the expression values in the 2 groups, with no differences seen in any of the measured inflammasome components or pro-cytokines (Figure 7B). To determine whether TNF has an effect on inflammasome oligomerization, we used immunofluorescence to visualize and quantify ASC specks from Nlrp $3^{3350 \mathrm{~V}} \mathrm{Cre}-\mathrm{ERT} 2$ and $\mathrm{Nlrp} 3^{\mathrm{A35OV}} \mathrm{Tnf}{ }^{\prime-}$ BMDCs and observed no significant differences before or after stimulation with LPS (Supplemental Figures 3 and 4). Similarly, we observed no significant differences in secreted mature IL-1 $1 \beta$ levels in cell supernatants (Supplemental Figure 3). Together, these data suggest that TNF is an important regulator at the transcriptional level for the NLRP3 inflammasome in murine CAPS. 

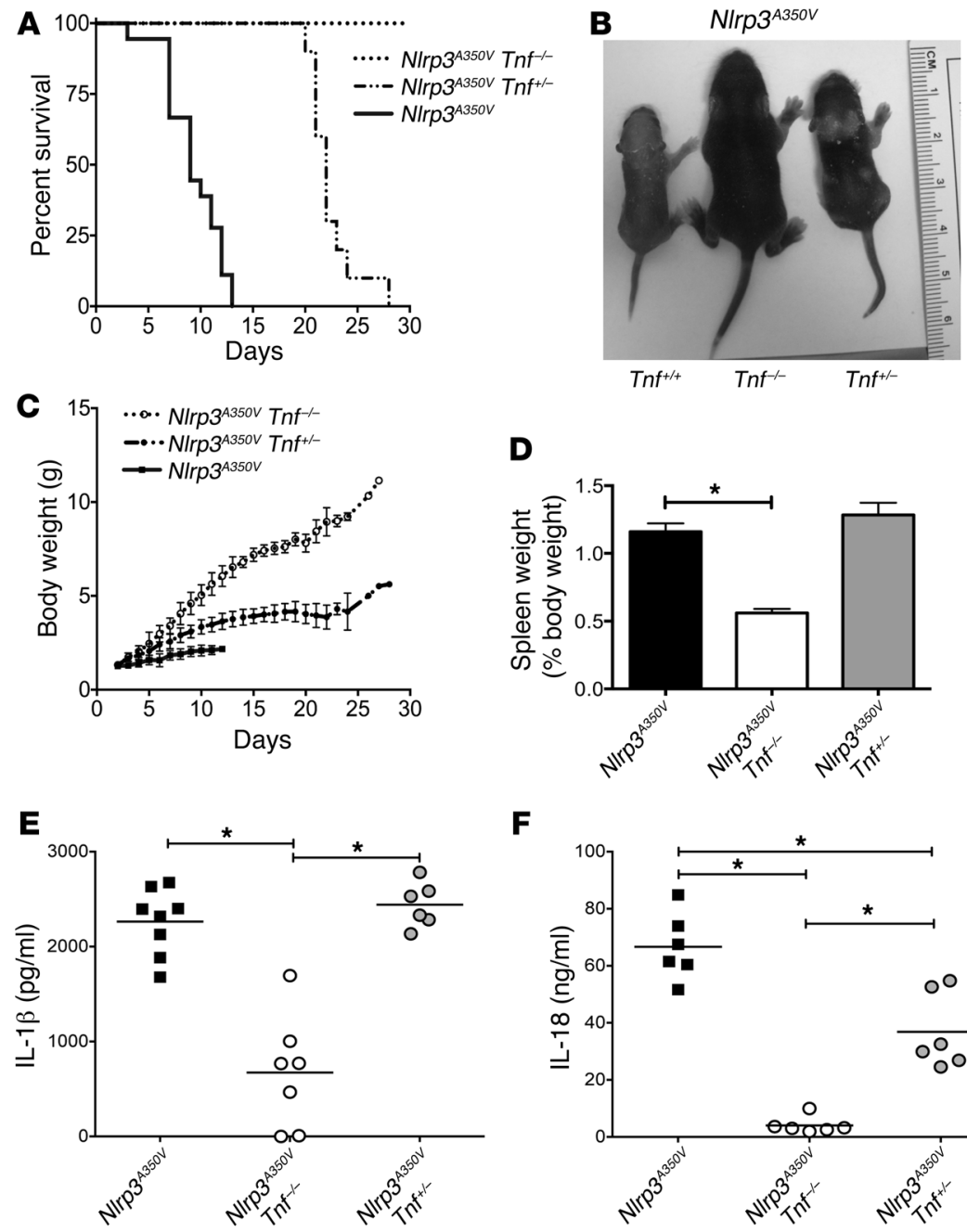

$\mathbf{F}$

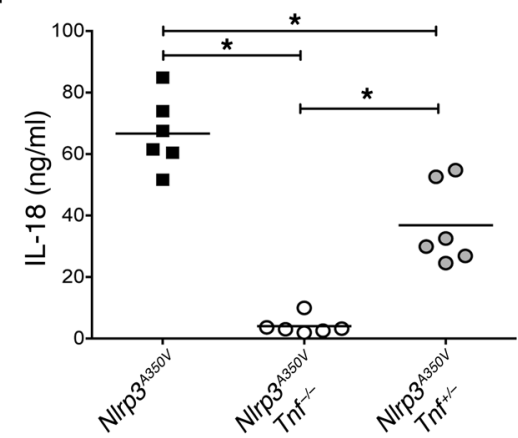

Figure 4. TNF deficiency prevents and attenuates systemic inflammatory disease in N/rp $3^{A 350 V}$ mice. (A-C) Survival and growth of $\mathrm{NIrp3}^{\mathrm{A350V}}, \mathrm{NIrp3}^{\mathrm{A} 350 \mathrm{~V}} \mathrm{Tnf}^{-/}$, and Nlrp3 ${ }^{\mathrm{A350V}} \mathrm{Tnf}^{+/-}$mice. Phenotypic comparison showing reduced survival and runting in N/rp $3^{A 350 V}$ mice, with complete and partial rescue in N/rp3 ${ }^{\mathrm{A350V}} \mathrm{Tnf}^{-{ }^{-}-}$and $\mathrm{Nlrp3}^{\mathrm{A35OV}} \mathrm{Tnf}^{+/-}$animals, respectively ( $n=6$ for N/rp3 $3^{\mathrm{A350V}}$ $\mathrm{Tnf}^{-/-}$mice, $n=10$ for N/rp3 $3^{\mathrm{A35OV}} \mathrm{Tnf}^{+/-}$mice, and $n=18$ for Nlrp $3^{A 350 V}$ mice). (D) The splenomegaly observed in NIrp3 ${ }^{\mathrm{A3} 30 \mathrm{~V}}$ and $\mathrm{Nlrp}^{\mathrm{A3} 30 \mathrm{~V}} \mathrm{Tnf}^{+/-}$mice was absent in Nlrp3 ${ }^{A 350 V}$ Tnf ${ }^{-1-}$ mice ( $n=5$ mice/group). ${ }^{*} P<0.05$ by Kruskal-Wallis with Dunn's multiple comparisons test. Data represent the mean \pm SEM. (E) Serum IL-1 $\beta$ levels were significantly reduced in $\mathrm{NIrp}^{\mathrm{A}}{ }^{\mathrm{350V}} \mathrm{Tnf}^{-/-}$mice compared with levels in NIrp3 ${ }^{A 350 V}$ mice, with no difference detected in N/rp3 ${ }^{\mathrm{A35OV}} \mathrm{Tnf}^{+/-}$mice. (F) N/rp3 $3^{\mathrm{A35OV}}$ $\mathrm{Tnf}^{-/-}$animals showed a significant reduction in serum IL-18 levels, while NIrp3 ${ }^{A 350 V} \mathrm{Tnf}^{+/-}$animals had an intermediate but significant reduction compared with levels in Nlrp3 ${ }^{A 350 V}$ mice. ${ }^{*} P<0.05$, by unpaired, 2-tailed Student's $t$ test (E and $\mathbf{F}$ ). Each data point represents an individual mouse; horizontal bars represent the mean.

\section{Discussion}

Recent studies of CAPS in humans and mice have advanced our knowledge of the NLRP3 inflammasome $(12,13)$ and the roles of both IL-1 $\beta$ and IL-18 in the subsequent inflammatory cascade (9). While translational studies have resulted in pivotal therapies for patients with CAPS $(14,15)$, many patients continue to have incomplete clinical responses, suggesting that our understanding of the inflammasome and other caspase-1-dependent pathways is incomplete. In this study, we took advantage of mutant Nlrp3-knockin mouse lines deficient in both IL-1 $\beta$ and IL-18 to investigate these alternate pathways.

Our studies demonstrated a clear caspase-1- and/or caspase-11-dependent inflammatory phenotype that was present in the Nlrp $3^{L 351 P} I_{l 1 b^{-/}} 1 l 18^{-/-}$mice as they aged. Although IL-1 $\beta$ and IL-18 both play prominent and distinct roles in murine disease pathology, residual inflammation remains, indicating that other mechanisms or inflammatory mediators are present. Here, we were able to show a significant role for TNF in the development and progression of murine inflammasomopathies through both genetic $\mathrm{KO}$ and targeted therapeutic treatment with etanercept, with either approach rescuing the early disease phenotype. We were further able to show a regulatory role for TNF at the level of pro-cytokine and inflammasome component mRNA expression.
Our discovery that serum levels of TNF were elevated in LPS-treated mutant mice in an inflammasome-dependent fashion, independent of IL-1 $\beta$ and IL-18, may be due to noncanonical inflammasome activation through caspase-11. This was described previously in LPS-induced sepsis models, but the responsible downstream cytokine was not identified (16). It is also possible that the elevated TNF levels in patients with NOMID and in the serum of mutant mice could be due to direct activation of NF- $\mathrm{BB}$ by the mutant NLRP3 inflammasome, a mechanism that has been demonstrated previously in some in vitro systems (17) but has not been shown conclusively in vivo. While inhibition of TNF in our KO models or via etanercept treatment demonstrates an important role for TNF in CAPS, it is not clear whether TNF is the primary driver of the remaining phenotype observed in our mice and in NOMID patients with an incomplete response to IL-1 blockade, or merely a key checkpoint in a complex inflammatory cascade.

TNF is a proinflammatory cytokine that is predominantly produced by activated macrophages to regulate the immune response. It acts as an endogenous pyrogen capable of inducing inflammation, apoptotic cell death, and cachexia. Consequently, dysregulation of TNF has been implicated in many inflammatory human diseases including psoriasis and inflammatory bowel 
A

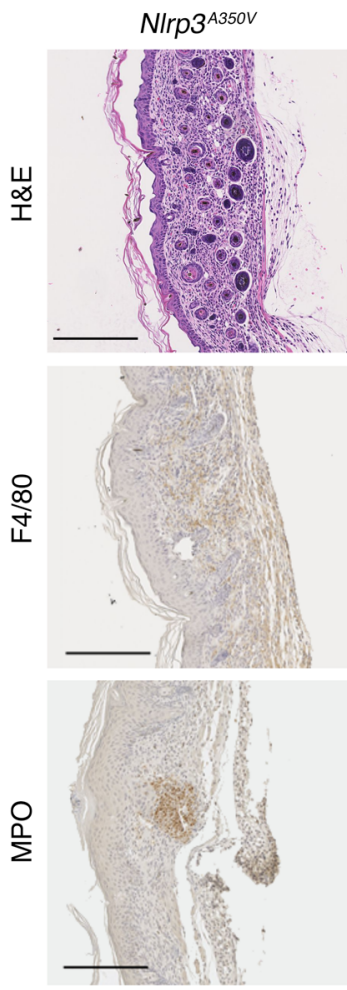

$\operatorname{Tnf}^{-1}$
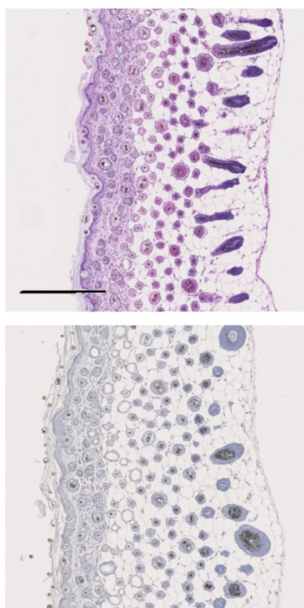

Nirp3 ${ }^{\text {A350V }}$ Tnf $^{-1}$
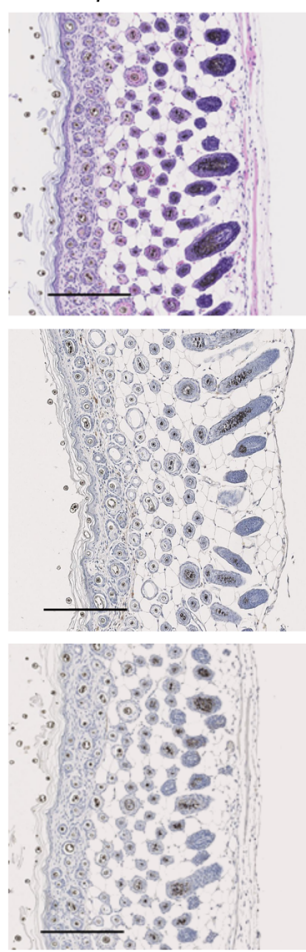

NIrp3 ${ }^{\text {A350V }}$ Tnf+- $^{+-}$

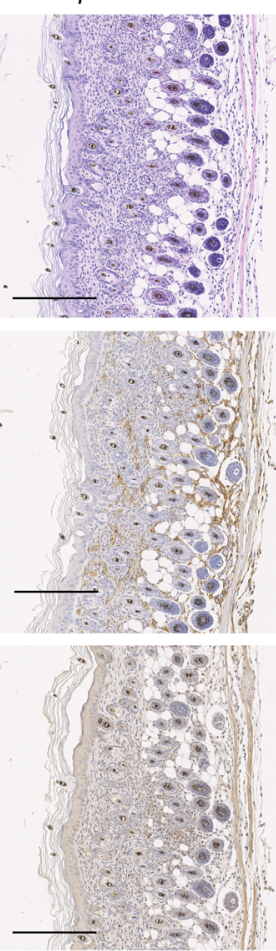

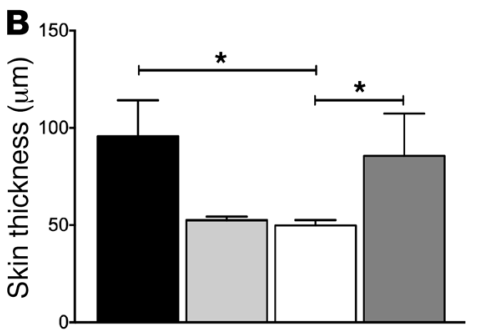
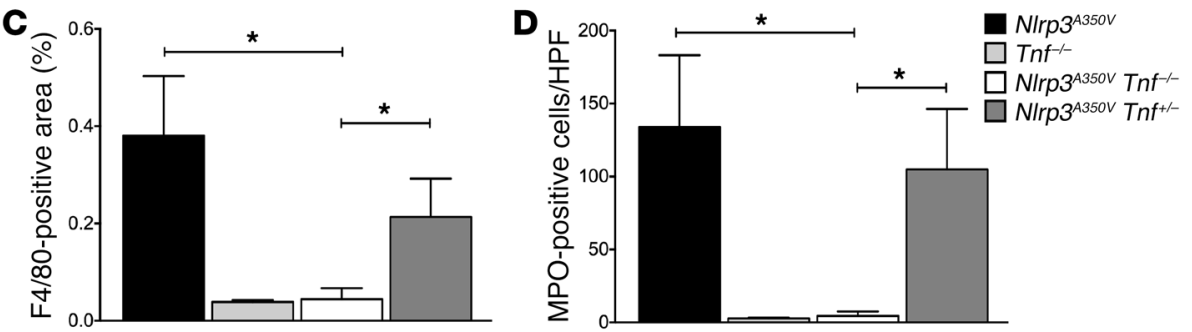

Figure 5. KO of TNF eliminates inflammatory skin disease in N/rp $3^{\mathrm{A35OV}}$ mice. (A-D) IHC of skin sections from N/rp $3^{\mathrm{A350V}}$ mice showed positive staining for F4/80 and MPO, with the presence of neutrophil pockets and a notable loss of s.c. tissue, while skin sections from N/rp $3^{\mathrm{A350V}} \mathrm{Tnf}^{-/-}$mice had a complete absence of these and were indistinguishable from those of control animals. N/rp3 ${ }^{\mathrm{A35OV}} \mathrm{Tnf}^{+/-}$mice were partially protected, with less $\mathrm{F} 4 / 80$ - and MPOpositive staining and increased s.c. tissue compared with skin tissue from N/rp3 $3^{A 350 V}$ mice. Quantification of (B) skin thickness, (C) F4/80-positive cells, and (D) MPO-positive cells. Representative sections and quantification of skin thickness and IHC are from 9 mice in each group. Representative sections in $\mathbf{A}$ are oriented with the basal region on the far right of each panel (original magnification, $\times 10$ ). Scale bar: $200 \mu \mathrm{m} .{ }^{*} P<0.05$, by Kruskal-Wallis with Dunn's multiple comparisons test. Data represent the mean \pm SEM.

disease $(18,19)$. TNF and IL-1 $\beta$ have been shown to strongly synergize in numerous biological functions, both in vitro and in vivo (20). Furthermore, TNF has been shown to induce IL-1 1 in human monocytes. TNF also enhances ATP- or silica-mediated caspase-1 and IL-1 $\beta$ secretion in murine macrophages and dendritic cells (21, 22). Although the effects of TNF and IL-1 $1 \beta$ overlap, they can have somewhat differing roles in the inflammatory cascade. In this study, we show significant decreases in both serum IL-1 $\beta$ and IL-18 levels as the result of TNF ablation, implicating the importance of TNF in inflammation and, in particular, the early autoinflammatory milieu in murine CAPS.

We were surprised to find a gene dosage-dependent effect in skin rash, inflammatory infiltrate, and survival kinetics in the Nlrp3 $3^{\mathrm{A35OV}} \mathrm{Tnf}^{+/-}$mice, in which an intermediate phenotype was observed between $N \operatorname{lrp} 3^{\mathrm{A35OV}}$ and $N \operatorname{lrp} 3^{\mathrm{A35OV}} \mathrm{Tnf}^{-}$animals. We did not observe this phenomenon in our previously generated mutant mice that were deficient for inflammasome components and cytokines $(8,9,23)$. This may be consistent with the regulatory role of TNF at the transcriptional level, which may allow for better fine-tuning of the inflammatory response.

The interplay between each of these proinflammatory cytokine pathways and the multistep regulation of inflammasome activity is likely to be complex and multifactorial. Our in vivo and in vitro data show significant reductions in splenic pro-cytokine mRNA levels alongside reductions in the upstream mRNA expression of the inflammasome components in $N \operatorname{lrp} 3^{3 \mathrm{ASOV}} \mathrm{Tnf} /$ - mice. This finding highlights the regulatory role of TNF in driving transcription of the cytokines and inflammasome components and may explain how targeting TNF in CAPS mice provides such a pronounced phenotypic rescue. 
A
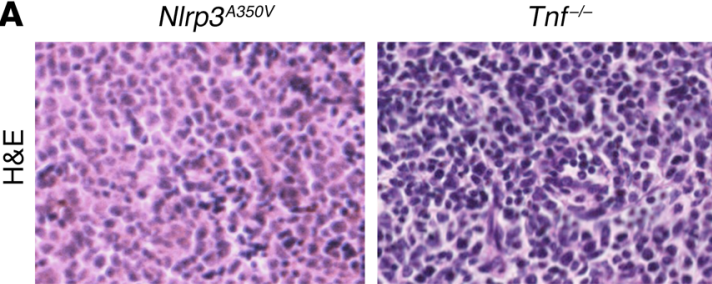

NIrp3 ${ }^{\mathrm{A350V}} \mathrm{Tnf}^{-/-}$

$\mathrm{Nlrp3}^{\mathrm{A} 350 \mathrm{~V}} \mathrm{Tnf}^{+-}$
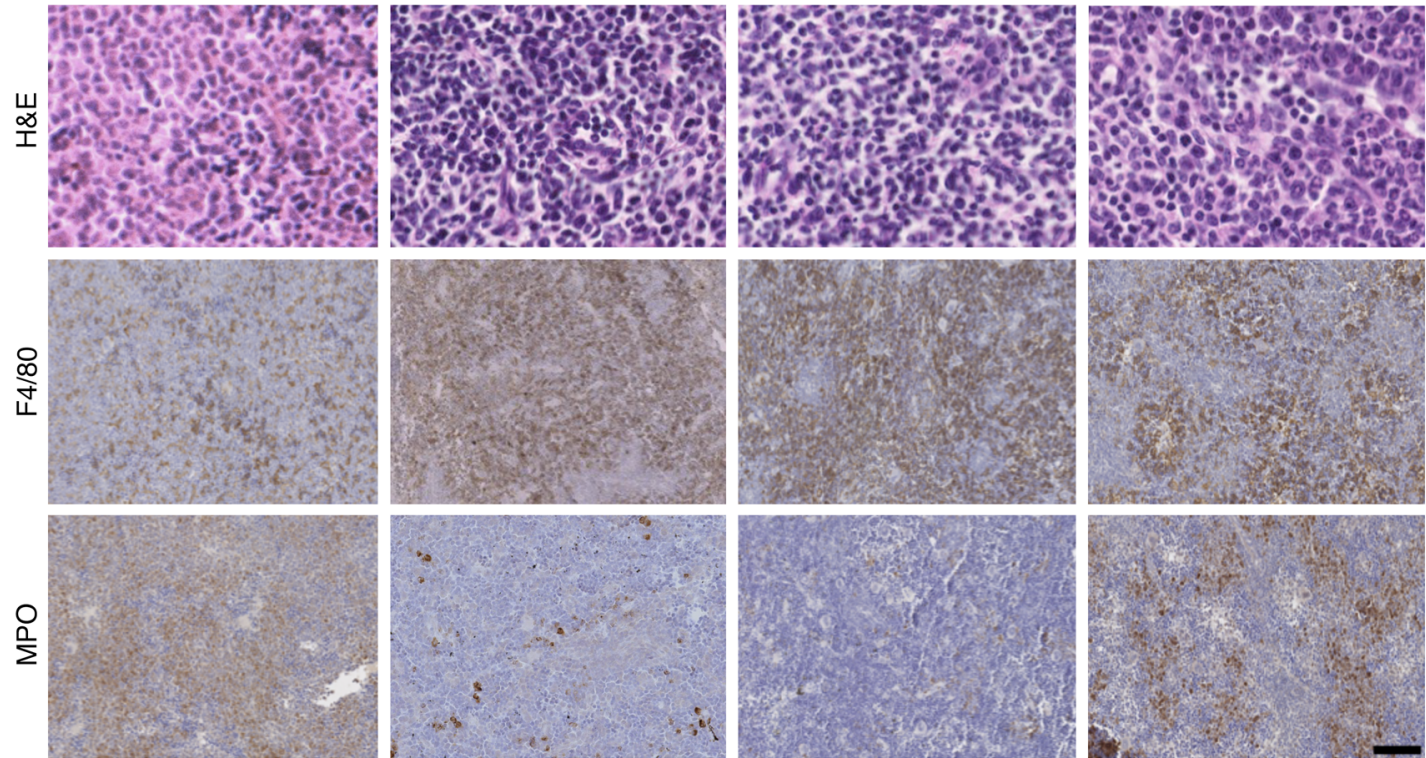

B
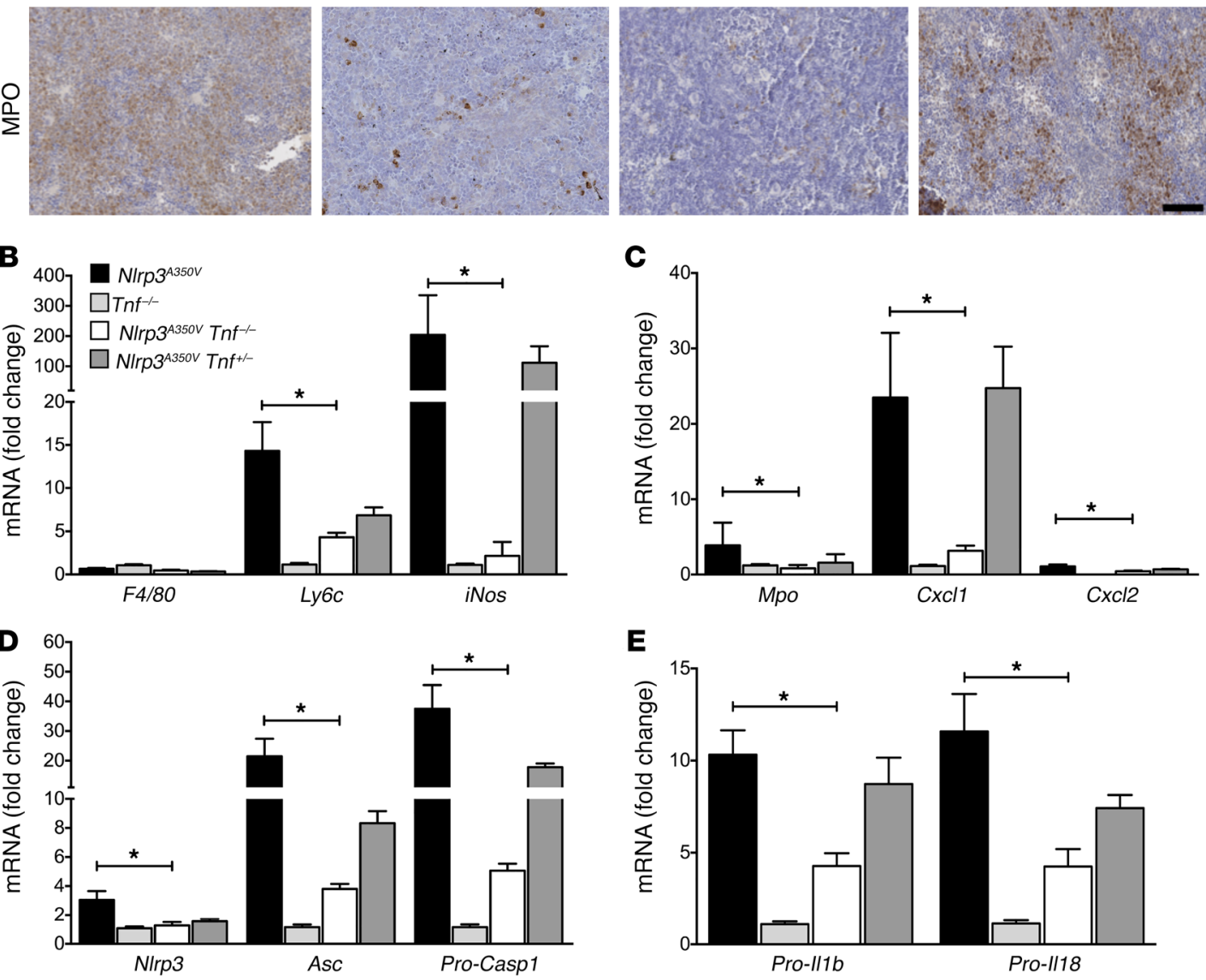

Figure 6. TNF ablation significantly reduces splenic myeloid infiltrate and expression of NLRP3 inflammasome components and pro-cytokines. (A) IHC

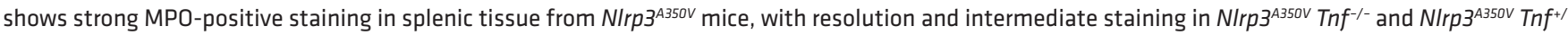
mice, respectively. Sections are representative of 6 mice per group (original magnification, $\times 40$ for H\&E staining and $\times 20$ for F4/80 and MPO staining). Scale bar: $100 \mu \mathrm{m}$. (B and C) Splenic mRNA levels of $\angle y 6 c$, iNos, Mpo, Cxcl1, and Cxc/2 were all significantly decreased in N/rp $3^{A 350 V} \mathrm{Tnf}^{-1-}$ animals compared with levels in intact-Nlrp3 ${ }^{\text {A350V }}$ mice. N/rp $3^{A 350 V} \mathrm{Tnf}^{+/-}$mice had a nonsignificant trend toward reduced $L y 6 c, M p o$, and $C x c / 2$ mRNA levels and similar values for iNos and $C x c / 1$ when compared with NIrp $3^{A 350 V}$ mice. (D and $\mathbf{E}$ ) Examination of inflammasome components revealed significant reductions in splenic mRNA expression of NIrp3, Asc, pro-Casp1, pro-II1, and pro- $/ 118$ in NIrp3 ${ }^{A 350 V} \mathrm{Tnf}^{-/-}$mice, with a trend toward reductions in all correlates in N/rp3 $3^{A 350 V} \mathrm{Tnf}^{+/-}$

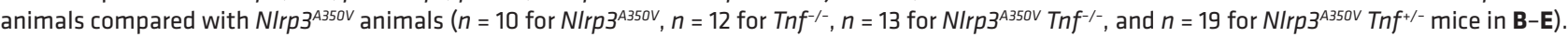
${ }^{*} P<0.05$, by Kruskal-Wallis with Dunn's multiple comparisons test. Data represent the mean \pm SEM.

Translating these findings, treatment of $N \operatorname{lrp} 3^{\mathrm{A} 350 \mathrm{~V}}$ pups with the TNF inhibitor etanercept was highly effective in normalizing both growth kinetics and systemic disease, even after the onset of inflammatory symptoms, as pups were born with skin abscesses that quickly cleared after beginning treatment on day 2 of life. A similar observation was made with an anti-TNF- $\alpha$-blocking $\operatorname{IgG}$ antibody treatment that predominantly affected the skin of a less severe knockin mutant mouse $\left(N l r p 3^{R 258 W}\right)(24,25)$. Given that some CAPS patients experience an incomplete clinical response to IL-1-targeted therapy and that some patients on therapy in our study had elevated serum TNF levels, the question remains whether select patients would benefit from TNF blockade in com- 

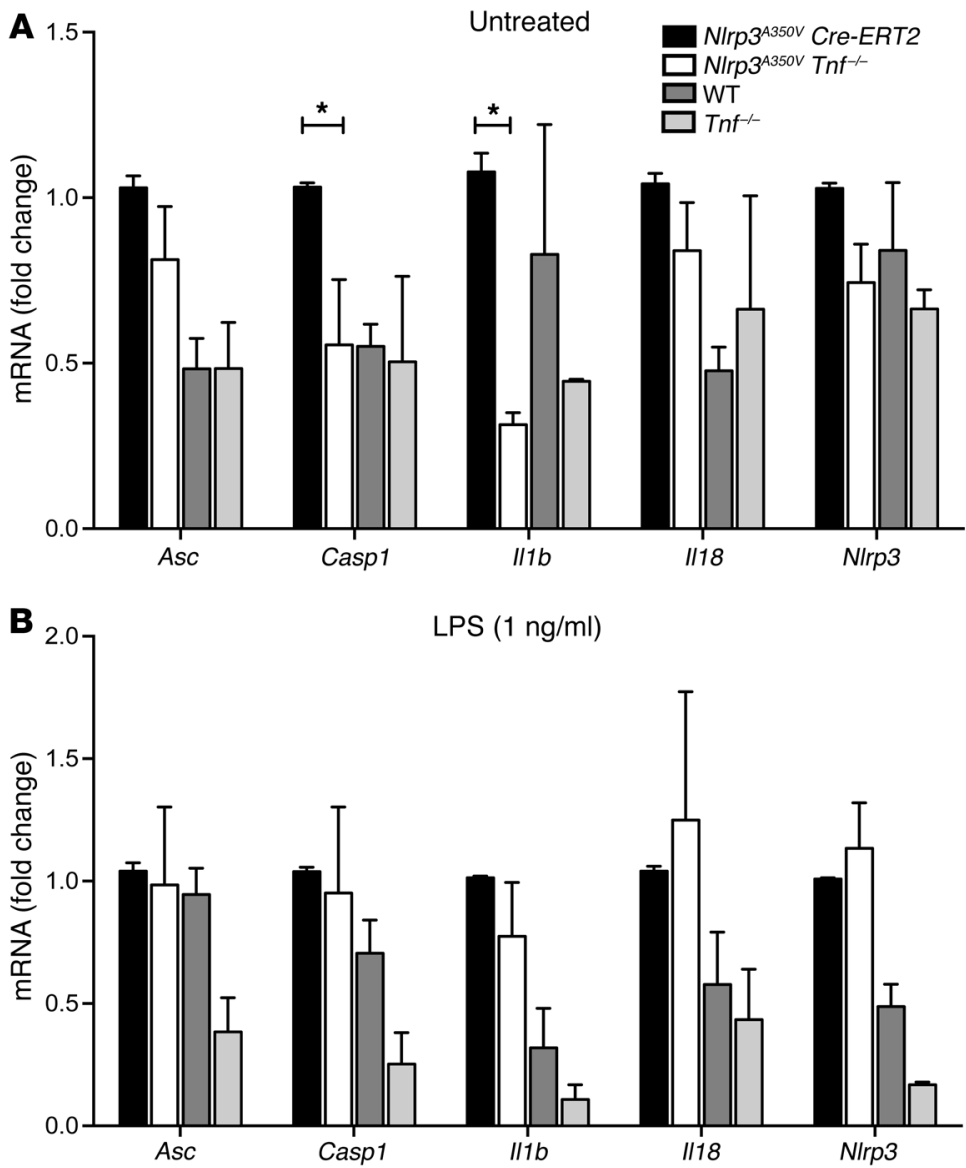

Figure 7. TNF KO in BMDCs significantly reduces baseline expression of pro-Casp1 and pro-II1b. (A) Cultured BMDCs from NIrp3 $3^{A 350 V} \mathrm{Tnf}^{-1-}$ mice had significantly reduced expression of pro-Casp1 and pro-II1b mRNA and similar levels for Nlrp3, Asc, and pro-I/18 when compared with expression levels in N/rp3 $3^{A 350 \mathrm{~V}}$ Cre-ERT2 BMDCs. (B) No significant differences were detected in the expression levels of inflammasome components or procytokines between Nlrp3 ${ }^{\text {A35OV }} \mathrm{Cre}-\mathrm{ERT2}$ and Nlrp3 ${ }^{\mathrm{A350V}} \mathrm{Tnf}^{-1-}$ BDMCs after stimulation with LPS $(1 \mathrm{ng} / \mathrm{ml}) . n=5$ mice per group for all measured values. ${ }^{*} P<0.05$, by 2 -way ANOVA with Dunn's multiple comparisons test. Data represent the mean \pm SEM.

bred with B6;129S-Tnftm1Gkl/J (Tnf ${ }^{-}$) mice (The Jackson Laboratory). Each of the mice described above were crossed with B6.129P2-Lyz2tm1(Cre)Ifo/J mice, allowing for the expression of mutant Nlrp3 in myeloid-derived cells in mice in which all tissues were deficient in Casp1/11, Illb/Il18, or $T n f$, respectively.

Complete blood count and ELISA. Complete blood count analysis was performed on $\mathrm{Nlrp} 3^{L 351 P}$ Casp1/11-/, Casp1/11-/, Nlrp $3^{2351 P} I l 1 b^{-1-} I l 18^{-/}$, and $I l 1 b^{-/} I l 18^{-/}$mice older than 6 months using a Scil Vet ABC Hematology Analyzer according to the manufacturer's instructions (Scil Animal Care Co.). Quantification of serum TNF, IL-1 $\beta$, and IL-18 levels in animals included in this study was also performed by ELISA according to the manufacturer's instruction (R\&D Systems).

In vivo LPS induction. Nlrp $3^{L 351 P}$ Casp1/11--, Casp1/11--,

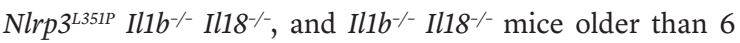
months were given a single i.p. injection of LPS from E. coli 0127:B8 (Sigma-Aldrich). LPS was injected at a concentration of $(5 \mu \mathrm{g} / \mathrm{g})$, and blood was collected by submandibular

bination with IL-1 inhibition. Prior to the use of IL-1 blockers in CAPS, there were unpublished reports of worsening symptoms in some CAPS patients treated with TNF blockers alone. Therefore, further studies are required to better understand the complex role that TNF plays in human CAPS pathology. Patients on combination therapy would also need to be monitored closely, as an increased infection rate was observed in rheumatoid arthritis patients treated with anakinra and etanercept together, without additional therapeutic benefit (26). However, the prominent regulatory role for TNF in inflammasome function and the significant reduction in murine CAPS pathology with TNF blockade make this a potential adjunctive therapeutic option in the future.

\section{Methods}

Mouse strains. Nlrp $3^{L 351 P n e o R}$ and $N \operatorname{lrp} 3^{\text {A350VneoR }}$ mice were generated as previously described, with leucine 351 to proline (L351P) and alanine 350 to valine (A350V) substitutions, respectively. The presence of an intronic floxed neomycin resistance cassette, in which expression of mutant Nlrp3 is restricted to cells of the myeloid lineage when bred into B6.129P2-Lyz2tm1(Cre)Ifo/J mice (LysM-Cre), generates Nlrp3 $3^{L 351 P}$ (perinatal lethality) and $N \operatorname{lrp} 3^{A 350 V}$-knockin mice (juvenile lethality) (The Jackson Laboratory) (8). Nlrp $3^{\text {L35IPneoR }}$ mice were bred with Casp $1 / 11^{-/-}$mice provided by R. Flavell (Howard Hughes Medical Institute, Yale University School of Medicine, New Haven, Connecticut, USA) or with $\mathrm{Illb}^{-/-} \mathrm{Il18^{-/- }}$ mice provided by E. Miao (Institute for Systems Biology, Seattle, Washington, USA) and D. Chaplin (University of Alabama, Birmingham, Alabama, USA). Nlrp $3^{\text {A350VneoR }}$ mice were puncture for serum cytokine analysis 1 day prior to and 2 and 6 hours after injection. For survival studies, LPS was injected at a concentration of $10 \mu \mathrm{g} / \mathrm{g}$, with survival analysis conducted every 3 hours, up to 24 hours after injection.

NOMID patients. As part of a longitudinal study of pediatric NOMID patients, blood was collected and serum analyzed from patients at baseline and during follow-up appointments at the National Institute of Arthritis and Musculoskeletal and Skin Diseases (NIAMS) Autoinflammatory Disease clinic (Bethesda, Maryland, USA), after beginning treatment on anakinra (Swedish Orphan Biovitrum $\mathrm{AB}$ [Sobi]), with doses ranging from 2.5 to $5.4 \mathrm{mg} / \mathrm{kg} / \mathrm{dl}$. Controls refer to pediatric patients who did not have an autoinflammatory condition. Patients' characteristics and clinical symptoms can be found in Supplemental Table 2.

Blood, spleen, and skin sample preparation. At the selected time interval, adult mice were anesthetized (ketamine $60 \mathrm{mg} / \mathrm{kg}$ plus xylazine $10 \mathrm{mg} / \mathrm{kg}$, i.p.), the peritoneal and thoracic cavities opened, and blood samples obtained via cardiac puncture. Neonates were sacrificed at 6 to 9 days of age and peripheral blood obtained by decapitation. The spleen and skin were harvested and the tissue was divided. A representative tissue section was fixed in 10\% formalin for 24 hours and embedded in paraffin, and 50- $\mu \mathrm{g}$ tissue samples were placed into 500 $\mu \mathrm{l}$ of RNAlater Solution (Life Technologies, Thermo Fisher Scientific).

Histology and IHC. For histological evaluation, splenic and skin tissues were stained with H\&E. The following primary monoclonal antibodies were used to perform immunostaining: anti-F4/80 (AbDSerotec/Bio-Rad; MCA497) and anti-MPO (Thermo Fisher Scientific; 
RB-373-A). Primary antibodies were omitted for the negative controls. The specimens were deparaffinized and rehydrated in ethanol and the antigens were retrieved in citrate buffer, $\mathrm{pH} 6.0$, for 30 minutes at $95^{\circ} \mathrm{C}$ and blocked with $2 \%$ BSA $1 \times$ Triton in TBS-T for 30 minutes at room temperature. Following overnight incubation with primary antibodies, a secondary antibody was applied and staining performed with a streptavidin-peroxidase complex, using 3,3-diaminobenzidine tetrahydroxychloride as DAB as chromogen. Slides were counterstained with hematoxylin. Photos were taken with a NanoZoomer 2.0HT Slide Scanning System (Hamamatsu) and/or microscope. A total of 3 fields per sample were examined. The number of MPO-positive cells as well as the F4/80stained areas per field were determined using Image J software (NIH).

Real-time PCR. Total RNA was isolated from splenic tissue and analyzed as previously described (3). The sequences of the primers used for qPCR are provided in Supplemental Table 1.

Etanercept treatment. Nlrp $3^{A 350 V}$ pups were treated s.c. with 400 $\mu \mathrm{g} / \mathrm{g}$ etanercept (Amgen) every other day, beginning on day 2 of life, for a total of 6 doses (27).

Cell culture and stimulation. In vitro stimulations were performed as previously described (8). Briefly, mouse BM cells were isolated from femurs and tibiae of Nlrp $3^{A 35 O V /+}$ Cre-ERT2 (conditional Nlrp3-mutant knockin mice in which the mutation is inducible with tamoxifen ex vivo) and $N \operatorname{lrp} 3^{A 350 V} \mathrm{Tnf}^{-/}$mice and plated in DMEM supplemented with $10 \%$ FCS, penicillin/streptomycin, L-glutamine, and sodium pyruvate $\left(4 \times 10^{5}\right.$ cells/well) in a 96-well plate with $20 \mathrm{ng} / \mathrm{ml} \mathrm{GM-CSF}(\mathrm{R} \& \mathrm{D}$ Systems) for 7 days, with 1 media change. BMDCs were then treated overnight with $1 \mu \mathrm{M}$ 4-hydroxy-tamoxifen (Sigma-Aldrich) to stimulate in vitro excision of neoR in Nlrp $3^{A 350 V /+}$ Cre-ERT2 cells. BMDCs were stimulated with or without $1 \mathrm{ng} / \mathrm{ml}$ LPS (Enzo Life Sciences; ALX-581010-L002) for 4 hours, and supernatants were collected.

Immunofluorescence staining and ASC specking. Cells were incubated in an 8-well chamber slide and stimulated with or without $1 \mathrm{ng} / \mathrm{ml}$ LPS for 4 hours. Cells were then washed, fixed with $4 \%$ PFA, blocked for 1 hour at room temperature, and incubated overnight with antiASC antibody (Enzo Life Sciences; ADI-905-173-100). The following day, cells were washed and incubated with Alexa 488 (Invitrogen, Thermo Fisher Scientific; A-21441) and DRAQ5 (Abcam; ab108410) for 1 hour at room temperature. ASC specks were imaged using a Leica confocal microscope at $\times 10$ magnification, with 6 images taken per well. Images were quantified using Image J to determine the total number of nuclei and specks. The total number of specks was added and divided by the total number of nuclei for all 6 images.

Statistics. Statistical analyses were performed using GraphPad Prism, version 5.03 (GraphPad Software). The significance level was set at $\alpha=5 \%$ for all comparisons. For experiments involving 3 or more groups, data were evaluated using Kruskal-Wallis with Dunn's multiple comparisons test, with the following exceptions: analysis of quantification of serum IL-1 $\beta$ and IL-18 levels was performed using an unpaired, 2-tailed Student's $t$ test, with comparisons made between all 3 groups, and analysis of in vitro BMDC experiments was performed using 2-way ANOVA with Dunn's multiple comparisons test, with comparison of Nlrp $3^{A 350 V} \mathrm{Cre}-E R T 2$ and Nlrp3A35OV Tnf ${ }^{--}$mice. Group comparisons were limited to 3 comparisons of particular inter-

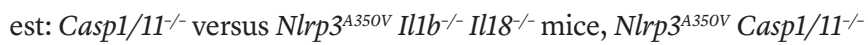

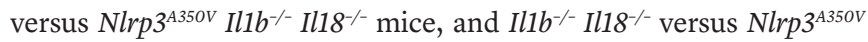
$I l 1 b^{-/-} \mathrm{Il} 18^{-/-}$mice, for the Illb/Il18-KO experiments; and Nlrp $3^{A 35 O V}$ ver-

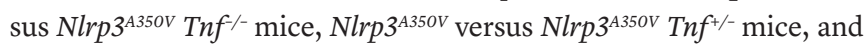
Nlrp3 $3^{A 350 V} \mathrm{Tnf}^{-/-}$versus Nlrp3 $3^{\mathrm{A} 350 \mathrm{~V}} \mathrm{Tnf} \mathrm{f}^{+-}$mice, for the Tnf-KO experiments. For experiments involving only 2 groups, a 2-tailed Student's $t$ test was used, except for comparisons of pre- and post-treatment measurements of serum from pediatric NOMID patients, in which a 1-tailed Student's $t$ test was used. A $P$ value of less than 0.05 was considered statistically significant. Unless otherwise stated, data are expressed as the mean \pm SEM or as the absolute number or percentage for categorical variables.

Study approval. The experimental protocols were approved by the IACUC of UCSD under protocol S04100. Studies involving patients were conducted in accordance with Declaration of Helsinki principles and the protocol for clinicaltrials.gov NCT00069329, and patients were recruited between September 22, 2003, and July 2014. The protocol was approved by the NIAMS institutional review board. Written informed consent was obtained from all patients or their legal guardians.

\section{Author contributions}

MDM, AW, RGM, LB, AEF, and HMH designed the research studies. MDM, AW, MEI, AH, CDJ, and CAP conducted the experiments. MDM, AW, MEI, RGM, LB, AEF, and HMH analyzed and interpreted the data. MDM, AW, LB, and HMH wrote the manuscript, which all the co-authors read and approved.

\section{Acknowledgments}

Funding was provided by NIH grant 1K08HD075830-01A1 (to LB); the German Research Foundation (DFG) (WR173/2-1 and SFB/TRR 57, to AW); the Intramural Research Program of the NIH, NIAID, and NIAMS (to RG); and NIH grants U01 AA022489 and R21AA023574 (to AEF) and R01 DK113592 (to AEF and HMH).

Address correspondence to: Hal Hoffman, 9500 Gilman Drive, Mail code 0760, La Jolla, California 92093-0760, USA. Phone: 858.534.2108; Email: hahoffman@ucsd.edu.
1. Martinon F, Burns K, Tschopp J. The inflammasome: a molecular platform triggering activation of inflammatory caspases and processing of proIL-beta. Mol Cell. 2002;10(2):417-426.

2. Bergsbaken T, Fink SL, Cookson BT. Pyroptosis: host cell death and inflammation. Nat Rev Microbiol. 2009;7(2):99-109.

3. Wree A, et al. NLRP3 inflammasome activation results in hepatocyte pyroptosis, liver inflammation, and fibrosis in mice. Hepatology. 2014;59(3):898-910.

4. Broderick L, De Nardo D, Franklin BS, Hoff- man HM, Latz E. The inflammasomes and autoinflammatory syndromes. Annu Rev Pathol. 2015;10:395-424.

5. Hoffman HM, Mueller JL, Broide DH, Wanderer AA, Kolodner RD. Mutation of a new gene encoding a putative pyrin-like protein causes familial cold autoinflammatory syndrome and Muckle-Wells syndrome. Nat Genet. 2001;29(3):301-305.

6. Aksentijevich I, et al. The clinical continuum of cryopyrinopathies: novel CIAS1 mutations in North American patients and a new cryopyrin model. Arthritis Rheum. 2007;56(4):1273-1285.
7. Meng G, Zhang F, Fuss I, Kitani A, Strober W. A mutation in the Nlrp3 gene causing inflammasome hyperactivation potentiates Th17 cell-dominant immune responses. Immunity. 2009;30(6):860-874.

8. Brydges SD, et al. Inflammasome-mediated disease animal models reveal roles for innate but not adaptive immunity. Immunity. 2009;30(6):875-887.

9. Brydges SD, Broderick L, McGeough MD, Pena CA, Mueller JL, Hoffman HM. Divergence of IL-1, IL-18, and cell death in aNLRP3 inflammasomop- 
athies. JClin Invest. 2013;123(11):4695-4705.

10. Rodriguez-Smith J, et al. Cerebrospinal fluid cytokines correlate with aseptic meningitis and blood-brain barrier function in neonatal-onset multisystem inflammatory disease: Central nervous system biomarkers in neonatal-onset multisystem inflammatory disease correlate with central nervous system inflammation. Arthritis Rheumatol. 2017;69(6):1325-1336.

11. De Filippo K, et al. Mast cell and macrophage chemokines CXCL1/CXCL2 control the early stage of neutrophil recruitment during tissue inflammation. Blood. 2013;121(24):4930-4937.

12. Carta $S$, et al. Cell stress increases ATP release in NLRP3 inflammasome-mediated autoinflammatory diseases, resulting in cytokine imbalance. Proc Natl Acad Sci U S A . 2015;112(9):2835-2840.

13. Coll RC, et al. A small-molecule inhibitor of the NLRP3 inflammasome for the treatment of inflammatory diseases. Nat Med. 2015;21(3):248-255.

14. Hoffman HM, et al. Prevention of cold-associated acute inflammation in familial cold autoinflammatory syndrome by interleukin-1 receptor antagonist. Lancet. 2004;364(9447):1779-1785. 15. Lachmann HJ, et al. In vivo regulation of interleu- kin 1 beta in patients with cryopyrinassociated periodic syndromes. JExp Med. 2009;206(5):1029-1036.

16. Kayagaki N, et al. Non-canonical inflammasome activation targets caspase-11. Nature. 2011;479(7371):117-121.

17. Manji GA, et al. PYPAF1, a PYRIN-containing Apaf1-like protein that assembles with ASC and regulates activation of NF-kappa B. J Biol Chem. 2002;277(13):11570-11575.

18. Victor FC, Gottlieb AB. TNF-alpha and apoptosis: implications for the pathogenesis and treatment of psoriasis. J Drugs Dermatol. 2002;1(3):264-275.

19. Brynskov J, et al. Tumour necrosis factor alpha converting enzyme (TACE) activity in the colonic mucosa of patients with inflammatory bowel disease. Gut. 2002;51(1):37-43.

20. Neta R, Sayers TJ, Oppenheim JJ. Relationship of TNF to interleukins. Immunol Ser. 1992;56:499-566.

21. Dinarello CA, et al. Tumor necrosis factor (cachectin) is an endogenous pyrogen and induces production of interleukin 1. JExp Med. 1986;163(6):1433-1450.

22. Franchi L, Eigenbrod T, Núñez G. Cutting edge: TNF-alpha mediates sensitization to ATP and silica via the NLRP3 inflammasome in the absence of microbial stimulation. JImmunol. 2009;183(2):792-796.

23. McGeough MD, et al. Cutting edge: IL- 6 is a marker of inflammation with no direct role in inflammasome-mediated mouse models. Jimmunol. 2012;189(6):2707-2711.

24. Nakamura Y, Franchi L, Kambe N, Meng G, Strober W, Núñez G. Critical role for mast cells in interleukin-1 $\beta$-driven skin inflammation associated with an activating mutation in the nlrp3 protein. Immunity. 2012;37(1):85-95.

25. Nakamura Y, Kambe N. Linkage of bacterial colonization of skin and the urticaria-like rash of NLRP3-mediated autoinflammatory syndromes through mast cell-derived TNF- $\alpha$. J Dermatol Sci. 2013;71(2):83-88.

26. Genovese MC, et al. Combination therapy with etanercept and anakinra in the treatment of patients with rheumatoid arthritis who have been treated unsuccessfully with methotrexate. Arthritis Rheum. 2004;50(5):1412-1419.

27. Zaid A, Rulli NE, Rolph MS, Suhrbier A, Mahalingam S. Disease exacerbation by etanercept in a mouse model of alphaviral arthritis and myositis. Arthritis Rheum. 2011;63(2):488-491. 